\title{
El primer paso: la pregunta clínica
}

\author{
JUAN CARLOS CLARO ${ }^{1,2,4}$, NICOLE LUSTIG ${ }^{1, \mathrm{a}}$, \\ MAURICIO SOTO ${ }^{1,3}$, GABRIEL RADA $^{1,2,4}$
}

\section{First step: the clinical question}

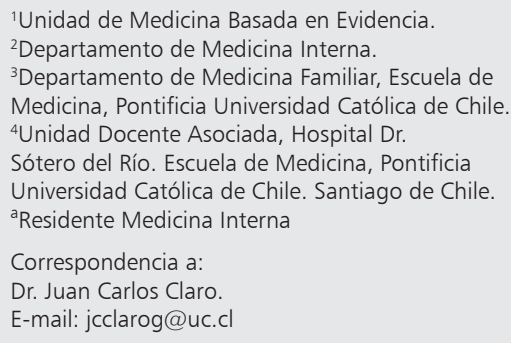

$\mathrm{E}$ n el año 2003 se inauguró en la Revista Médica de Chile una Sección titulada "Medicina Basada en Evidencia" (MBE). En concordancia con el ciclo de la MBE, uno de los primeros artículos publicados en dicha Sección se centró en la formulación de una pregunta clínica adecuada ${ }^{1}$. Desde entonces hasta ahora, ha habido un desarrollo importante en el ámbito de la MBE, siendo oportuno actualizar los conceptos planteados en aquel artículo.

Durante la práctica clínica es frecuente que frente a diversas situaciones surjan preguntas, tanto por parte del clínico como del propio paciente. Estas preguntas pueden ser de índole práctica como, por ejemplo ¿dónde retiro mis medicamentos? o ¿de cuántos miligramos son los comprimidos de metformina que entregan en el consultorio? Por otro lado, existe otro tipo de preguntas, relacionadas más directamente con la situación clínica concreta, y que podemos clasificar en dos grupos:

Preguntas de preparación (background): forman parte del conocimiento general de una condición. Habitualmente se responden en libros de texto y artículos de revisión (revisión narrativa; el tradicional review de muchas revistas médicas). Buscan responder aspectos relacionados con la naturaleza de dicha condición, del tratamiento o métodos diagnósticos habituales de una enfermedad (p. ej. ¿cuáles son las alternativas de tratamiento para una neumonía adquirida en la comunidad, en adultos?, ¿cuáles son los métodos diagnósticos disponibles para evaluar un abdomen agudo en mujeres en edad fértil?).

Preguntas de acción (foreground): son aquellas preguntas específicas que nos formulamos para tomar decisiones clínicas en distintos ámbitos como, por ejemplo: acerca de los efectos de terapias, exactitud o impacto de métodos diagnósticos u observaciones acerca de la causa de algunas enfermedades. Estas preguntas constituyen la base de la práctica de la Medicina Basada en Evidencia (p. ej. ¿Es útil la amoxicilina para el tratamiento de la rinosinusitis aguda en adultos?, ¿cuál es el riesgo de presentar hipoglicemias nocturnas con insulina glargina (ultra-lenta) comparado con insulina lenta, en pacientes diabéticos?). Este tipo de preguntas más avanzadas o específicas sólo podrían ser contestadas utilizando los resultados de estudios clínicos que hayan sido realizados en grupos de pacientes de las mismas características y con problemas similares. Conforme un médico va avanzando en la práctica de la profesión va cambiando la proporción de preguntas de preparación y de acción que se hace. De este modo, inicialmente la mayoría de las preguntas son de preparación, y muy pocas son de acción, mientras que cuando va obteniendo experiencia y conocimiento, surgen preguntas más específicas o de acción (Figura 1).

Es importante destacar que, a pesar de tener una vasta trayectoria clínica y amplios conocimientos, nunca dejan de generarse preguntas de preparación. Nadie había oído hablar hasta hace pocos años del SARS (síndrome respiratorio agudo severo), y por tanto, todas las preguntas que esta condición generó inicialmente fueron de preparación, incluso para los más experimentados especialistas.

\section{¿Qué y cuántas preguntas clínicas se hace un profesional de salud?}

Diversos estudios han intentado responder a esta pregunta. Una revisión de la literatura encontró que profesionales de salud que trabajaban en atención primaria se hacen 0,07 a 1,85 preguntas por cada paciente que consulta. Este margen fue atribuido a diferentes escenarios clínicos, diferen- 
El primer paso: la pregunta clínica - J. C. Claro et al

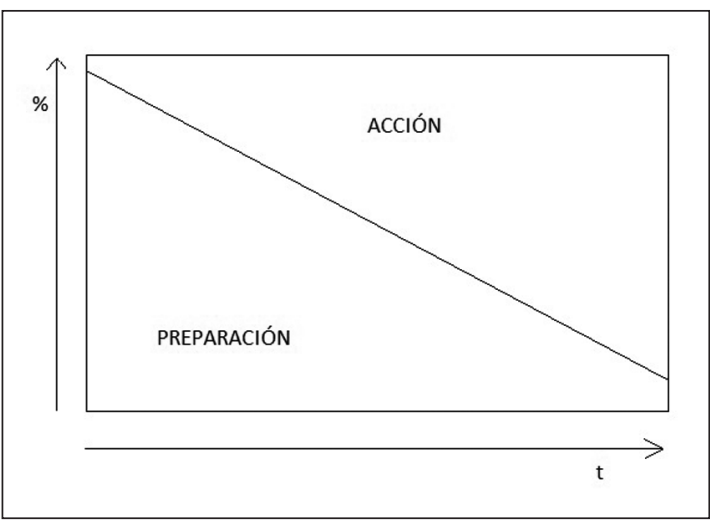

Figura 1. En el eje $X$ se representa el tiempo (t) en el que se desarrolla nuestra experiencia clínica. En el eje $Y$ el total de preguntas que nos hacemos. Al inicio de nuestra carrera clínica, la mayoría de las preguntas que nos hacemos son de preparación (background), y conforme aumenta nuestra experiencia, aumenta la proporción de preguntas de acción (foreground) aunque nunca llegan a desaparecer las de preparación.

cias en la definición de pregunta o en el método de evaluación usado. Entre 30\% y 56\% de esas preguntas fue seguido de una búsqueda de información, lo cual fue más probable si el problema era urgente o si era alta la expectativa de que existiese una respuesta clara ${ }^{2}$.

En otro estudio se evaluaron las preguntas de un grupo de pediatras y médicos de familia ${ }^{3}$. Las preguntas de diagnóstico fueron entre 50 y $60 \%$ del total, las de terapia entre 20 y $30 \%$, y las no clínicas o de índole práctica cerca del 15\%.

Por otro lado, en un estudio realizado en un Servicio de Medicina de un Hospital universitario, por cada 10 pacientes hospitalizados, se generaban 57 preguntas por parte del tutor y los alumnos (de pre y postgrado), siendo la mayoría (74\%) acerca del cuidado directo de los pacientes ${ }^{4}$. Esto sugiere que los ambientes docente-asistenciales tienden a generar más preguntas por paciente que los espacios puramente asistenciales.

\section{¿Por qué estructurar la pregunta clínica de acción?}

Las preguntas clínicas pueden ser muy frecuentes y generarse a partir de variadas situaciones, tanto médicas como no médicas.

Transformar un problema clínico en una pregunta clínica es la primera etapa en la práctica de

\section{Tabla 1. Etapas en la práctica de la Medicina} Basada en Evidencia

1. Convertir el problema clínico en una pregunta bien formulada

2. Buscar eficientemente la mejor evidencia que la responda

3. Realizar un análisis crítico de la evidencia encontrada

4. Hacer uso de lo encontrado y analizado en la toma de decisiones

la Medicina Basada en Evidencia ${ }^{1}$ (Tabla 1). Sin embargo, antes de empezar a buscar respuestas es conveniente que nuestra pregunta sea formulada de tal forma que nos permita delimitar, al máximo posible, las necesidades de información y nos facilite la elaboración de una estrategia de búsqueda eficiente.

Para ayudarnos en este proceso se han planteado las características que debe cumplir una pregunta clínica bien elaborada ${ }^{5}$ :

1. Definir claramente el problema a resolver.

2. Facilitar la estrategia de búsqueda.

3. Definir el tipo de evidencia y, por tanto, el diseño de estudio más adecuado para poder responderla de la mejor manera.

En otras palabras, es conveniente evitar, en la medida de lo posible, búsquedas demasiado amplias, mediante la construcción de preguntas clínicas bien formuladas.

\section{Componentes de una pregunta de acción}

Las preguntas clínicas de acción pueden hacer referencia a distintos ámbitos. Habrá ocasiones en que nos preguntaremos acerca de los efectos de determinada intervención en comparación a otra, en una patología específica y con un objetivo concreto. En otras nos preguntaremos sobre la exactitud de determinado examen de laboratorio para el diagnóstico de una condición, o incluso acerca del rol de la exposición a cierto factor de riesgo para el desarrollo de una enfermedad. Dentro de la literatura se han definido los componentes que debe tener una pregunta clínica estructurada, inicialmente para preguntas de terapia, pero posteriormente para los otros ámbitos (diagnóstico, pronóstico, etc. $)^{6}$ : 
P: Población: Corresponde a las personas a las que se va a aplicar la información encontrada.

Incluiremos aquellas características clínicamente importantes que están relacionadas con la intervención, es decir, aquellas que si están presentes o ausentes modifican la respuesta. Por ejemplo: si nuestra paciente presenta hipertensión arterial y un cáncer de mama en etapa inicial, este último es muy relevante clínicamente. Sin embargo, si nos preguntamos cuál es el efecto del enalapril en esta paciente, sólo nos interesa el primero (no existe ninguna razón fisiopatológica para pensar que el enalapril actúe distinto en mujeres hipertensas con o sin cáncer de mama). Visto de otra forma, son aquellas características que un investigador consideraría como criterios de inclusión para un estudio clínico (es prácticamente impensable que un investigador restrinja sus criterios para incorporar pacientes a un estudio que evalúa los efectos del enalapril a mujeres con cáncer de mama).

Es conveniente intentar reducir la definición de población al mínimo posible de características (en la gran mayoría de los casos basta un término para realizar una buena definición de Población). A medida que se agregan más conceptos, la posibilidad de encontrar evidencia disminuye. Esto obedece a razones conceptuales (no todos los estudios utilizan criterios de inclusión tan estrictos), a razones relacionadas con cierto tipo de evidencia (las revisiones sistemáticas (RS) suelen buscar preguntas amplias, para luego abordar los casos más específicos o subgrupos) y a razones prácticas (la indización en base de datos no siempre considera todos los términos posibles).

A: Acción: Si bien todos los componentes son importantes para formular una buena pregunta clínica, la acción que se evalúa es el único componente imprescindible en una pregunta de acción (valga la redundancia). En el caso de las preguntas de terapia, esta acción es la Intervención cuyos efectos se evalúan. En la enseñanza de la metodología de la Medicina Basada en Evidencia, en particular cuando el espacio curricular no permite abordar todos los ámbitos de la pregunta clínica, se suele privilegiar la enseñanza de la metodología en torno a la toma de decisiones terapéuticas, en cuyo caso, se suele sustituir la A (acción) por la I (intervención). Al igual que otros autores, nos parece preferible utilizar el concepto Acción, ya que las decisiones clínicas no se circunscriben sólo al ámbito terapéutico ${ }^{7}$. De hecho, las preguntas de diagnóstico pueden ser incluso más frecuentes que las de terapia ${ }^{3}$.

C: Comparación: En el caso de las preguntas de terapia, corresponde a la alternativa contra la cual la intervención se debiera comparar. En casos en que la intervención es la única opción terapéutica existente para determinada condición, la comparación ideal es el tratamiento óptimo (estándar, habitual, rutinario) complementado con un placebo, con el fin de minimizar el sesgo durante el desarrollo del estudio ${ }^{8}$. Si existen alternativas eficaces en uso, la comparación óptima corresponde al mejor tratamiento disponible. Existe mucha controversia sobre la utilidad (y la justificación ética) de estudios que comparan contra placebo cuando existe una terapia efectiva9.

O: Objetivo: Es lo que se busca conseguir con la acción. En el caso de intervenciones terapéuticas, se trata del desenlace o evento (outcome o endpoint). Es fundamental diferenciar outcomes importantes para el paciente, de outcomes intermedios. Estos últimos son variables o parámetros que supuestamente predicen el outcome importante para el paciente, lo cual no necesariamente se cumple en todos los $\operatorname{casos}^{10,11}$.

\section{Ámbito de la pregunta de acción}

Revisemos los componentes recién mencionados, aplicados a los diferentes ámbitos de preguntas de acción:

1. Preguntas de terapia: Si bien el concepto de "terapia" excluye a las intervenciones preventivas, en este caso nos referimos a "intervenciones preventivas o terapéuticas". Hemos preferido mantener el nombre "terapia" por ser de amplio uso y fácil conceptualización. Los aspectos relacionados con este ámbito han sido discutidos en la sección anterior para ejemplificar los distintos componentes de una pregunta; Población, Acción (intervención), Comparación (placebo u otra comparación activa), Objetivo (desenlace o outcome).

El estudio que responde a esta pregunta de manera más confiable (menor riesgo de sesgo) es el Estudio Controlado Randomizado (ECR), por lo tanto, la evidencia que se debe buscar para responder a estas preguntas son Revisiones Sistemáticas (RS) de ECRs.

2. Preguntas de diagnóstico: Dentro del proceso que realizamos para poder llegar a un diagnóstico, 
se utilizan diversos elementos que van modificando las probabilidades ${ }^{12}$, como síntomas, signos, exámenes de laboratorio, imágenes, etc. Para cada uno de estos "tests", se pueden plantear preguntas a distintos niveles.

a) Preguntas de exactitud diagnóstica (diagnostic accuracy): Se compara el test diagnóstico con el gold standard para evaluar cuán exacto es este nuevo test para hacer diagnóstico de una condición determinada. La Población corresponde a pacientes en que se sospecha el diagnóstico, la Acción es el test diagnóstico, la Comparación es el gold standard, y el Objetivo es la correcta identificación de la condición buscada, la cual se suele expresar en términos de sensibilidad, especificidad, e idealmente likelihood ratio $^{13}$. El estudio primario más confiable para responder a estas preguntas es un estudio de corte transversal en que a los pacientes se les aplican ambos test; el que está en evaluación y el gold standard ${ }^{14}$. La mejor evidencia corresponde a una RS de este tipo de estudios, las cuales se denominan habitualmente RS de exactitud diagnóstica.

b) Preguntas de utilidad diagnóstica (diagnostic utility): Buscan evaluar el impacto que un determinado test diagnóstico tiene sobre un determinado objetivo en una población. Por ejemplo: en adultos jóvenes con cuadro de abdomen agudo, el uso de un TAC de abdomen al ingreso, comparado con la conducta quirúrgica habitual (que no incluye TAC), ¿disminuye la tasa de laparotomías en blanco? En este caso, la Población y Acción son similares a las preguntas de exactitud diagnóstica, pero el Objetivo es impactar sobre outcomes clínicos (Tabla 2).

El estudio más confiable para responder esta pregunta es un ECR en que en la población en estudio, a un grupo se le realice un TAC y el otro reciba la atención habitual (cirugía u observación), para evaluar finalmente si la aplicación de dicho test sirvió para disminuir la tasa de laparotomías en blanco. Una RS de ECR nos permitirá conocer la mejor evidencia para responder a este tipo de pregunta.

3. Preguntas de pronóstico: Se formulan en individuos que ya presentan una determinada condición, en los que se desea por un lado estimar su probable curso clínico en un período de tiempo determinado o, por otro, evaluar el efecto que determinadas exposiciones tienen sobre dichos pacientes. Por ejemplo:

- En pacientes hospitalizados que ingresan por hemorragia digestiva alta y vuelven a sangrar durante la hospitalización, ¿cuál es su riego de morir a 1 mes?

- En adultos mayores con antecedentes de accidente vascular encefálico, ¿cuál es su riesgo de convulsiones a 1 año?

- En mujeres con cáncer de mama, la presencia de un determinado polimorfismo genético, comparado con la ausencia del mismo, ise asocia menor sobrevida?

En los dos primeros ejemplos no se aplica una comparación, sino que lo que queremos evaluar es la ocurrencia (eventualidad) de determinada complicación en un período de tiempo, mientras que en el tercer ejemplo nos enfocamos en el efecto que tiene determinada exposición en la condición de dichos pacientes (Tabla 3 ).

Los estudios para responder a estas preguntas son observacionales, siendo en general el de cohorte prospectiva el más confiable. Cuando la incidencia del evento es muy baja, pueden ser preferibles los estudios de casos y controles. Una RS de estudios observacionales constituye la mejor evidencia en este ámbito.

4. Preguntas de riesgo o daño: Existe un grupo de preguntas que evalúan cuán importante es un

Tabla 2. Ejemplos de preguntas de diagnóstico

\begin{tabular}{|c|c|c|c|c|}
\hline Elemento & $\mathbf{P}$ & A (Test) & C & 0 \\
\hline $\begin{array}{l}\text { Exactitud (accuracy) } \\
\text { (de un test) }\end{array}$ & $\begin{array}{l}\text { En pacientes adultos } \\
\text { mayores, con cuadro } \\
\text { de cólico renal... }\end{array}$ & $\begin{array}{l}\text {... el uso de una eco- } \\
\text { grafía de abdomen }+ \\
\text { sedimento de orina... }\end{array}$ & $\begin{array}{l}\text {... comparado con } \\
\text { un PieloTAC (gold } \\
\text { standard)... }\end{array}$ & $\begin{array}{l}\text {... ¿identifica correcta- } \\
\text { mente la urolitiasis? }\end{array}$ \\
\hline Utilidad (de un test) & $\begin{array}{l}\text { En pacientes adultos } \\
\text { jóvenes, con cuadro } \\
\text { de abdomen agudo... }\end{array}$ & $\begin{array}{l}\ldots \text { el uso de TAC de } \\
\text { abdomen... }\end{array}$ & $\begin{array}{l}\text {... comparado con } \\
\text { manejo quirúrgico } \\
\text { habitual... }\end{array}$ & $\begin{array}{l}\text {... ¿disminuye la tasa } \\
\text { de laparotomías en } \\
\text { blanco? }\end{array}$ \\
\hline
\end{tabular}


El primer paso: la pregunta clínica - J. C. Claro et al

Tabla 3. Ejemplos de preguntas de pronóstico

\begin{tabular}{|c|c|c|c|c|}
\hline Elemento & $\mathbf{P}$ & A (exposición) & C & 0 \\
\hline $\begin{array}{l}\text { Eventualidad } \\
\text { (likelihood) }\end{array}$ & $\begin{array}{l}\text { En pacientes con hemo- } \\
\text { rragia digestiva alta... }\end{array}$ & $\begin{array}{l}\text {... que resangran } \\
\text { durante la hospitaliza- } \\
\text { ción... }\end{array}$ & (no se aplica) & $\begin{array}{l}\text {... ¿cuál es la mortali- } \\
\text { dad? }\end{array}$ \\
\hline $\begin{array}{l}\text { Efecto de la } \\
\text { exposición }\end{array}$ & $\begin{array}{l}\text { En mujeres con cáncer } \\
\text { de mama... }\end{array}$ & $\begin{array}{l}\text {... la presencia de } \\
\text { determinado polimor- } \\
\text { fismo genético... }\end{array}$ & $\begin{array}{l}\text {... comparado con } \\
\text { la ausencia de dicho } \\
\text { polimorfismo... }\end{array}$ & $\begin{array}{l}\text {... ¿aumenta la mor- } \\
\text { talidad? }\end{array}$ \\
\hline
\end{tabular}

factor determinado en una población para el desarrollo de una determinada condición o evento. Por ejemplo:

- ¿Tienen los fumadores mayor riesgo que los no fumadores para desarrollar cáncer bronquial?

- Los pacientes con urolitiasis sometidos a radiografías seriadas en el seguimiento de su cuadro, ¿tienen más probabilidad de desarrollar leucemia aguda que pacientes no irradiados?

Si bien estas preguntas podrían formar parte de las preguntas de terapia, diagnóstico o pronóstico, vale la pena separarlas, dado que su objetivo es evaluar el desarrollo de una condición nociva para el paciente. Es por esto mismo que las respuestas las podemos encontrar en estudios tan diversos como ECR que reporten daño como efecto adverso de la intervención en estudio, o en estudios observacionales.

En el primer caso, la Acción está constituida por la exposición a determinado factor. En general se utiliza el concepto Daño para las consecuencias indeseadas de las intervenciones médicas (por ej.los efectos adversos de un medicamento) y Riesgo para la exposición a otros factores (por ej. el tabaco).

\section{Conclusión}

A partir del encuentro clínico con cada paciente se pueden generar múltiples dudas. Con cada una de ellas comienza un nuevo ciclo en la práctica de la medicina basada en evidencia.

Es importante convertir estas dudas en preguntas clínicas bien formuladas. Para ello, debemos reconocer cuáles corresponden a preguntas de acción, reconocer el ámbito al que pertenece cada pregunta, diferenciar sus distintos componentes, y tener en cuenta cuál es el diseño de estudio más confiable para responderla (una revisión sistemática de todos esos estudios constituirá la mejor evidencia). Todo esto permitirá realizar una adecuada estrategia de búsqueda.

El éxito del proceso propuesto por la $\mathrm{MBE}$ depende de manera crítica de una pregunta clínica bien formulada.

\section{Referencias}

1. Soto M, Rada G. Formulación de preguntas en Medicina Basada en Evidencia. Rev Med Chile 2003; 131: 1202-3.

2. Coumou HC, Meijman FJ. How do primary care physicians seek answers to clinical questions? A literature review. J Med Libr Assoc 2006; 94: 55-60.

3. González-González AI, Sánchez Mateos JF, Sanz Cuesta T, Riesgo Fuertes R, Escortell Mayor E, Hernández Fernández T. Estudio de las necesidades de información generadas por los médicos de atención primaria (proyecto ENIGMA). Aten Primaria 2006; 38: 219-24.

4. Osheroff JA, Buchanan BG, Bankowitz RA, Blumenfeld $\mathrm{BH}$, Miller RA. Physicians' information needs: analysis of questions posed during clinical teaching. Ann Intern Med 1991; 114: 576-81.

5. Mayer D. What is evidence-based medicine? En: Mayer D (editor). Essential Evidence-Based Medicine. Cambridge, UK: Cambridge University Press;2010. p. 14-5.

6. Straus SE. Formular preguntas clínicas contestables. En: Straus SE, Richardson WS, Glasziou P, Haynes B (editores). Medicina basada en evidencia. Cómo practicar $y$ enseñar la MBE (versión en español de la 3ra edición de la obra original en inglés "Evidence-Based Medicine"). Madrid, España: Elsevier; 2006. p. 16-7.

7. Silva SA, Charon R, Wyer PC. The marriage of evidence and narrative: scientific nurturance within clinical practice. J Eval Clin Pract 2011; 17: 585-93.

8. Letelier LM, Manríquez JJ, Claro JC. ¿El “ciego” en los ensayos clínicos importa? Rev Med Chile 2004; 132: 1137-9.

9. Michels KB, Rothman KJ. Update on unethical use of 
placebos in randomised trials. Bioethics 2003; 17 (2): 188-204.

10. Guyatt G, Montori V, Devereaux PJ. Patients at the center: in our practice, and in our use of language. ACP J Club 2004; 140 (1): A11-2.

11. Neumann I, Claro JC. Outcomes intermedios. Rev Med Chile 2012; 141 (1): 113-6.
12. Capurro D, Rada G. El proceso diagnóstico. Rev Med Chile 2007; 135: 534-8.

13. Salech F, Mery V, Larrondo F, Rada G. Estudios que evalúan un test diagnóstico: interpretando sus resultados. Rev Med Chile 2008; 136: 1203-8.

14. Valenzuela DL, Cifuentes AL. Validez de estudios de tests diagnósticos. Rev Med Chile 2008; 136: 401-4. 